\title{
Pixelization and dynamic range in radio interferometry
}

\author{
W. D. Cotton and J. M. Uson
}

\author{
National Radio Astronomy Observatory ${ }^{\star}, 520$ Edgemont Road, Charlottesville, VA 22903-2475, USA \\ e-mail: bcotton@nrao.edu
}

Received 19 November 2007 / Accepted 5 September 2008

\begin{abstract}
This study investigates some of the consequences of representing the sky by a rectangular grid of pixels on the dynamic range of images derived from radio interferometric measurements. In particular, the effects of image pixelization coupled to the CLEAN deconvolution representation of the sky as a set of discrete delta functions can limit the dynamic range obtained when representing bright emission not confined to pixels on the grid. Sky curvature effects on non-coplanar arrays will limit the dynamic range even if strong sources are centered on a pixel in a "fly's eye" representation when such pixel is not located at the corresponding facet's tangent point. Uncertainties in the response function of the individual antennas as well as in the calibration of actual data due to ionospheric, atmospheric or other effects will limit the dynamic range even when using grid-less subtraction (i.e. in the visibility domain) of strong sources located within the field of view of the observation. A technique to reduce these effects is described and examples from an implementation in the Obit package are given. Application of this technique leads to significantly superior results without a significant increase in the computing time.
\end{abstract}

Key words. techniques: image processing - techniques: interferometric

\section{Introduction}

With the new generation of high sensitivity interferometers to come on-line in the next few years (EVLA, ALMA, LOFAR) wide-field imaging will be necessary to achieve the sensitivity possible with these instruments. The problem is especially acute at low frequencies $(<10 \mathrm{GHz})$ where every field of view will contain several relatively bright sources at any time. The sensitivity of instruments such as LOFAR or the EVLA at lower frequencies may be compromised much of the time by artifacts due to the bright sources in the field if these artifacts are not reduced to an acceptable level. This paper describes artifacts arising from using pixelated images to describe the sky as well as a technique for reducing them. All data manipulations discussed in this report used the Obit package (Cotton 2008, http: //www . cv.nrao.edu/ bcotton/Obit.html).

\section{Effects of pixelated images}

It is generally convenient to represent the sky seen by an imaging interferometer as a set of pixel values on a rectangular grid. This is a good match to the widely used CLEAN deconvolution technique which represents the sky as a set of delta functions located at the centers of cells on such a grid. A commonly used measure of the quality of an image is its "dynamic range," generally defined to be the ratio of the brightest pixel in an image to the rms pixel-to-pixel fluctuation in areas devoid of emission. Application of this criterion is generally straightforward as the response of the primary beam leads to mostly empty regions in the images sufficiently far from the pointing center. This convention is adopted in the following.

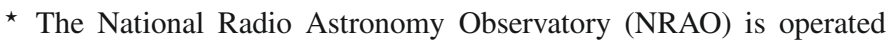
by Associated Universities Inc., under cooperative agreement with the National Science Foundation.
One limitation of the pixelization technique is that emission not confined to points on the grid cannot be represented exactly and the CLEAN technique will approximate such structure by a potentially infinite series of alternating positive and negative delta functions. The problem is particularly severe when the image contains very bright, unresolved emission as is common in the radio sky. A combination of the limited support of the actual representation and the effects of the finite precision of digital computers will limit the dynamic range obtained by introducing artifacts in the derived image. This effect has long been recognized as a problem (Briggs \& Cornwell 1992; Perley 1999a). Briggs \& Cornwell (1992) describe the result as due to the discontinuity in the visibility function of an off-center point source at the boundary of the sampled region of the $u-v$ plane which requires an infinite number of components in the image plane to model it accurately. Briggs \& Cornwell (1992) estimate that this effect will limit dynamic range to $\approx 1000$.

This effect is easily understood for an unresolved source located between pixels. In order to model a point source between pixels, the deconvolution must add emission in the adjacent pixels. To counteract the resultant broadening of the source, negative emission must then be added around the source. An example of this effect is clearly shown in Fig. 1 which compares the results of CLEANing a model point source both exactly centered on a pixel and offset between pixels. For the offset source, the off-source rms is $2.3 \times 10^{-4}$ of the model source flux density (dynamic range $=4300$ ) while for the centered source, the offsource rms is $4.7 \times 10^{-10}$ of the model source flux density (dynamic range $=2.1 \times 10^{9}$ ). In the latter case, the result is limited by the precision of 32-bit digital arithmetic.

In the past, uncentered point sources have not been a particularly serious problem as the bright source was usually the object under investigation and centering it on a pixel was straightforward. This is not the case for surveys or future observations where the source(s) of interest may be faint sources 

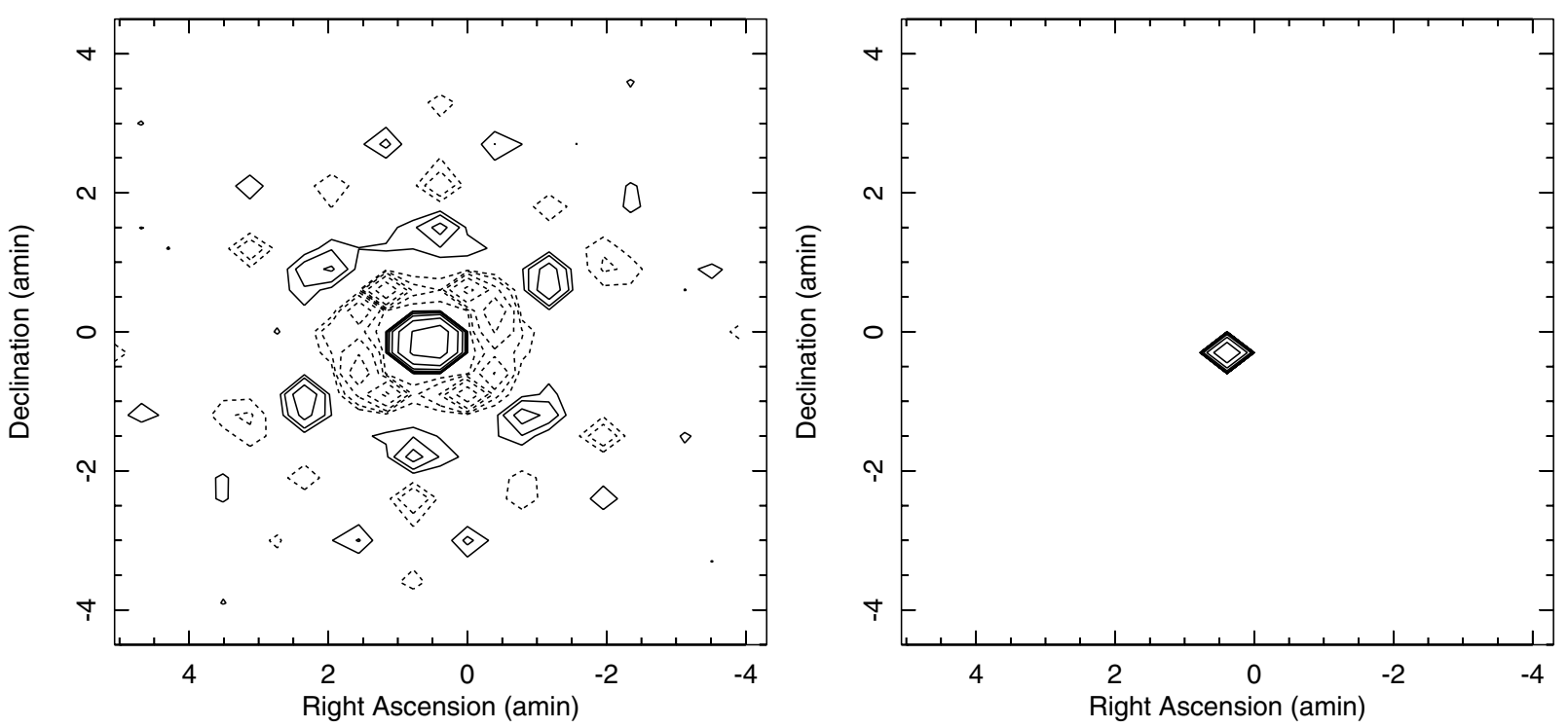

Fig. 1. Contour plots of example CLEAN images derived from noiseless point source model data restored using delta functions for the components to demonstrate the actual distribution of locations of CLEAN components. The plots show the same region and have the same contour levels, factors of powers of $2 \times 0.1 \%$ of the model flux density; negative contours are dashed. Left: the point source was located approximately midway between cells in both dimensions. CLEANing used 1000 components. Right: the point source was located exactly on the central pixel. Only the central pixel contains emission; CLEANing used 200 components.

in the presence of multiple, much stronger sources whose locations on the imaging grid are not easily controlled. Processing of the NRAO VLA Sky Survey (NVSS) (Condon et al. 1998) used partial pixel shifts to align the imaging grid to a single exceptionally bright $(>0.5 \mathrm{Jy}$ ) source whenever such a source was present in the field.

A second limitation of the pixelization technique is that the rectangular grid is flat whereas the sky is not, see Cornwell \& Perley (1992). In the case of an array confined to a plane during synthesis, such as the $E-W$ linear Westerbork Synthesis Radio Telescope, a projection of the sky is possible which avoids this problem; but, in the general case, this is not possible. This effect is referred to in the following as the co-planarity problem. If the field of interest is small enough, the curvature of the sky can be negligible. Several techniques have been developed to solve the more general problem. Some of these are:

- Full 3-D imaging: interferometric measurements are made in visibility space described by the coordinate set $(u, v, w)$. These measurements can be convolved onto a 3D grid and Fourier transformed into a 3D image. The celestial sphere is a spherical surface in this 3D image. A 3D deconvolution followed by projection onto a plane is possible but in practice this is sufficiently expensive in computing resources that it is not used. See Perley (1999b) for details.

- Fly's Eye: the curved surface of the celestial sphere can be approximated by a mosaic of facets, each tangent to the celestial sphere and of sufficiently limited extent that the error introduced is negligible. However, the errors increase quadratically with the distance of a cell to the corresponding tangent point and this can still limit the dynamic range. See "the Polyhedron Method" in Cornwell \& Perley (1992) for details.

- $W$ projection: it is possible to correct for the diffractive effects on the wavefront as it propagates from the antenna closer to the source to the farther on each baseline. This correction is made to the convolution of the visibility data onto the grid prior to Fourier transformation. This allows a single, flat 2D grid to represent the curved sky. However, the derived "dirty" image is no longer strictly a convolution. See Cornwell et al. (2005) for details.

In the following, only adaptations of the Fly's Eye technique are considered.

\section{Wide-field imaging with Fly's Eye and catalog of sources}

The technique used for wide-field imaging in the following tests is as follows. A circular field of view to be completely imaged is specified by its radius from the pointing center. The data are examined and the cell spacing (if not specified) is picked on the basis of the longest baseline in the data (one quarter of the smallest fringe spacing) and the size of a facet "undistorted" by co-planarity effects determined from the maximum extent of any baseline in the selected data in the direction of the pointing center. The radius of an undistorted region is adapted from Thompson (1999) as $0.33 \sqrt{1 / \max W}$ radians where $\max W$ is the maximum value of the " $w$ " in the data set in wavelengths. A "Fly's Eye" tessellation of circular regions in a hexagonal pattern is then defined which fully covers the field of view and a set of square images enclosing these circular regions defines a mosaic of facets.

In general, the imaged field of view does not enclose all of the sky to which the array elements have significant gain, so that sources are visible outside of this fully-imaged field of view. To include such sources, facets are added to the mosaic centered on the positions of outlying sources obtained from a catalog (currently a stripped-down version of the NVSS) which are deemed to have an apparent brightness above a given userspecified threshold based on an assumed spectral index and a model of the antenna gain pattern. These additional facets do not need to be contiguous with those covering the field of view wanted. At high frequencies, a catalog could be generated from the WMAP catalog of point sources (Bennett \& WMAP team 2007). Accurate positions and flux densities are not required, 
only a list of positions around which a bright source might appear, as the decision to auto-center is based on the results of the initial CLEAN.

The variant of CLEAN used in the following discussion is the "visibility based" or "Cotton-Schwab" CLEAN (Schwab 1983; Cotton 1989), in which the Fourier transform of the estimate of the sky is iteratively subtracted from the visibility data and the residual image re-derived. This allows multiple independent "facets" to be imaged on the sky. All facets in the mosaic to be deconvolved are formed and a quality measure based on both peak (residual) brightness and extended emission $(0.95 \times$ peak absolute value residual in the clean window $+0.05 \times$ the average residual - as used in the AIPS package ${ }^{1}$ ) is used to determine which facet is to be CLEANed next. Components are selected from this facet and then subtracted from the visibility data. The next highest quality measure facet is re-imaged and, if it still maintains its status, it is CLEANed; otherwise, the next highest facet is re-imaged and tested, etc. The CLEAN is stopped when one of two conditions is satisfied, 1) the total number of CLEAN components reaches a user specified limit or; 2) the maximum absolute value residual in the CLEAN window of all facets is less than a user specified minimum, usually of order of the anticipated rms in the image.

After CLEAN is finished, components are (optionally) convolved with a Gaussian approximation to the instrumental PSF and restored to the facet from which they were subtracted as well as to any overlapping facet containing their positions. After restoration of the subtracted components, all facets are projected ("flattened") onto a grid covering the specified field of view.

Because strong "flanking sources" can be observed with dedicated pointings prior to reducing the data at hand and can thus be available in a catalog, it would seem possible (in principle) to subtract them from the measured visibilities prior to processing of the target field. However, such visibility-based subtraction is hindered by foreground effects on the calibration as well as by imprecise knowledge of the response of the primary beam of the antennas which modify the apparent position and flux density of the cataloged sources. Efforts to fit these effects in the uv-plane have had limited success. The technique has been shown to work reasonably well for only a few sources using simulated data and appears to require vast computing resources to handle even a few sources (Voronkov \& Wieringa 2004).

\section{AutoCenter technique}

In order to minimize pixelization related artifacts using the Fly's Eye technique, it is desirable to locate each strong, point-like source exactly on the pixel that is at the tangent point of the facet that contains it. This is accomplished by first identifying these sources in an initial imaging step and then adding facets for strong sources not already very close to a facet center. It is also necessary to restrict CLEAN from assigning any components to any overlapping regions of other facets.

An initial CLEAN is used to determine which objects in the field, if any, have sufficiently bright emission to warrant being centered on a special facet. The CLEAN needs to be deep enough that an accurate measure of the centroid of the source can be made to subsequently center it on a pixel. Currently, the implementation in the Obit package CLEANs to a factor of 0.1 of the user-specified auto-center brightness threshold above which artifacts are expected to be above the noise level. The initial CLEAN level needed depends on the uv coverage, and dynamic

1 http://www.aips.nrao.edu range but generally a factor of 100 to 1000 below the peak is adequate. Also, since the residuals are not needed, it is not necessary to derive a full set of residual images at the end of the initial CLEAN. The decision that AutoCentering is needed is based on the highest pixel value in the CLEAN-able portions of the initial dirty images.

Peak brightnesses are determined from the sum of the CLEAN components within a given radius (cells within $62.5 \%$ of the FWHM of the PSF in the current implementation) of each component derived from each facet. If this sum exceeds the autocenter brightness threshold level, then the centroid of the peak is determined from the CLEAN components by a moment analysis. A small facet, currently $96 \times 96$ pixels centered at the derived centroid, is added to the working imaging mosaic. It is possible that the same source may appear in several overlapping facets in the initial fly's eye tiling so it is necessary to ensure that a given strong source is added only once, a coincidence of better than one-half of the FWHM of the synthesized beam (typically two cells) is considered to correspond to the same source. If a source is already within 0.5 pixel of the tangent pixel of the enclosing facet, a new facet is not created, but the facet is re-centered (if not already within 0.01 cell).

In order to ensure that CLEAN assigns no components to the re-centered source appearing in overlapping facets of the initial mosaic, each of the facets is examined to see if it contains the position of the source to be re-centered. In any facet in which the re-centered source appears in the CLEAN-able region, a round "unbox", currently of radius 33 pixels, is added to that facet centered on the position of the corresponding source. This size is slightly smaller than the size of the initial cleanable region in the new facet added, a radius of 38 pixels, to ensure that no pixel will be excluded a priori from a component search given the non-coplanarity of the original and the new facets. These choices are somewhat arbitrary but seem to work well. An "unbox" is like a normal CLEAN window except that any enclosed pixel will not be considered as a location for CLEAN components even if located inside of another regular CLEAN box, i.e. the CLEAN process ignores any pixels inside of an unbox. Pixels inside of unboxes are also excluded from statistical estimates such as maximum, minimum and rms. If any re-centering operations are required, the initial CLEAN is repeated. After this re-centering, all prior CLEAN components are discarded before beginning a CLEAN.

Because the accuracy of the determination of the centroid of a source to be re-centered is adversely affected by the limited CLEAN and the very effects this technique is trying to correct, some iteration may be in order. Images in which high dynamic range is desired generally benefit as well from one or more iterations of self-calibration. At the beginning of each CLEAN, the centroids of auto-centered sources from the previous CLEAN are checked to see if they are sufficiently close to the tangent pixel. If the first (reduced) moment of CLEAN components within 1.5 pixels of the central pixel is offset by more than 0.01 pixel, then the image is re-centered and all components from the previous CLEAN are discarded. This allows an iterative refinement of the centroid of the peak and improves significantly the results over a single estimate.

\section{Examples}

The following sections give two sets of examples processed using this technique. The first involves simulated data and the second, actual VLA observations of 3C 84. 
Table 1. Offset source dynamic ranges.

\begin{tabular}{ccc}
\hline \hline Offset(pixels) & DR $^{a}$ & DR_corr $^{b}$ \\
\hline 0 & $>1.0 \times 10^{23}$ & $>1.0 \times 10^{23}$ \\
0.01 & $260 \times 10^{3}$ & $9.2 \times 10^{6}$ \\
0.02 & $130 \times 10^{3}$ & $10.1 \times 10^{6}$ \\
0.03 & $94 \times 10^{3}$ & $10.3 \times 10^{6}$ \\
0.05 & $60 \times 10^{3}$ & $8.9 \times 10^{6}$ \\
0.1 & $29 \times 10^{3}$ & $8.9 \times 10^{6}$ \\
$0.141(0.1,0.1)^{c}$ & $17 \times 10^{3}$ & $4.9 \times 10^{6}$ \\
0.2 & $17 \times 10^{3}$ & $11.5 \times 10^{6}$ \\
$0.283(0.2,0.2)^{c}$ & $9.7 \times 10^{3}$ & $4.4 \times 10^{6}$ \\
0.3 & $12 \times 10^{3}$ & $10.2 \times 10^{6}$ \\
0.5 & $10 \times 10^{3}$ & $9.6 \times 10^{6}$ \\
$0.707(0.5,0.5)^{c}$ & $6.2 \times 10^{3}$ & $7.1 \times 10^{6}$ \\
\hline 10 & $65 \times 10^{3}$ & $69.6 \times 10^{6}$ \\
20 & $16 \times 10^{3}$ & $79.1 \times 10^{6}$ \\
30 & $7.3 \times 10^{3}$ & $66.3 \times 10^{6}$ \\
50 & $2.7 \times 10^{3}$ & $8.1 \times 10^{6}$ \\
100 & $0.74 \times 10^{3}$ & $0.4 \times 10^{6}$ \\
\hline
\end{tabular}

Notes: ${ }^{a}$ Dynamic range without autoCenter; ${ }^{b}$ dynamic range with autoCenter; ${ }^{c}$ offsets along both coordinates.

\subsection{Simulated data}

Simulated data have the advantage that their properties are known which makes it simple to separate artifacts from source structure. In these tests, the model used was a single $1 \mathrm{Jy}$ point source with no noise or other corruptions added. In order to test the effects of fractional pixel offsets, the source was located at the phase center of the data and the data imaged with a series of fractional pixel shifts added to the center of the image. The artificial data set used the same uv-plane sampling as a $74 \mathrm{MHz}$ VLA data set which consisted of 12 frequency channels of $122 \mathrm{kHz}$ bandwidth. The tests were performed in the Obit package. The CLEAN used the visibility-based technique and proceeded for 1000 iterations with a loop gain of 0.1 and a CLEAN window of radius 10 cells centered on the peak. The model visibility computation used the "DFT" method which is more accurate than the "GRID" method as implemented in Obit (and AIPS). Several iterations of imaging/re-centering were done in order to refine the estimate of the centroid. The cell spacing used was 0.25 of the FWHM of the fitted Gaussian restoring beam so the image was reasonably over-sampled. The components removed by the CLEAN procedure were not restored and the rms of the pixel values in the final residual image was used to derive the dynamic range. Each of the tests was then repeated turning on the autoCenter mode and the corresponding dynamic range determined in turn. A combination of offsets on a single axis and on both axes are included. The results are shown in Table 1 and Fig. 2.

A second set of tests explored the effects of non co-planarity by inserting a series of large, whole-pixel offsets to the position of the point source model and using a procedure like the one described above for small pixel offsets. The results are shown in Table 1 and Fig. 3. All offsets except the one of 100 pixels are within the "undistorted field of view" of a facet.

While these tests are not exhaustive, it is clear that fractional pixel offsets of bright point-like sources can limit the dynamic range to $\sim 10^{4}$ and non co-planar effects can limit the dynamic range to $\sim 10^{3}$ even with a moderately conservative limit on facet size. Applying the autoCenter technique improved the dynamic range by typically a factor of 50 to 1000 for the fractional pixel

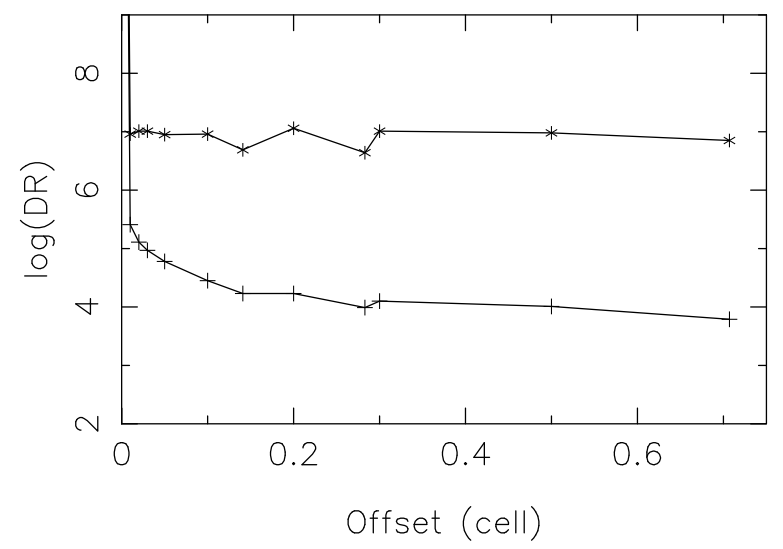

Fig. 2. Dynamic Range (DR) obtained on simulated, noiseless data as a function of fractional pixel offset. A combination of offsets on one and both axes are included. The "+" symbols represent CLEANing without using autoCenter and the "*” represent the results of using autoCenter.

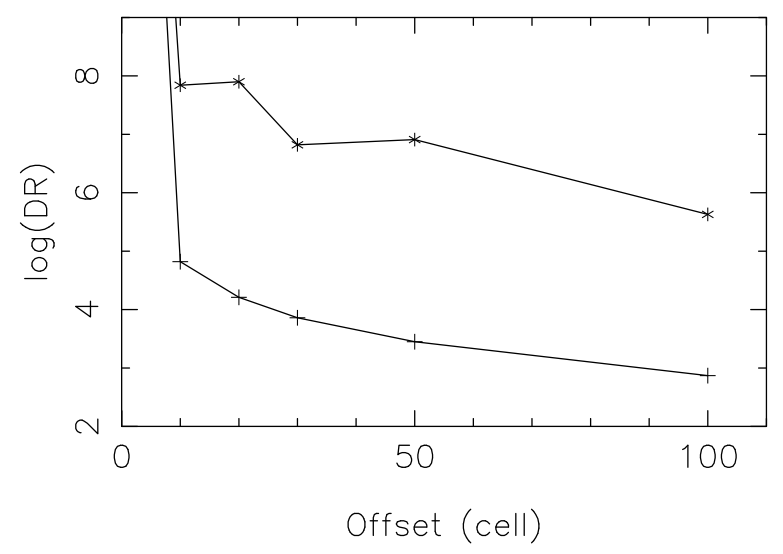

Fig. 3. Dynamic Range (DR) obtained on simulated, noiseless data as a function of large, whole pixel offsets to test non co-planarity effects. The "+" symbols represent CLEANing without using autoCenter and the " $*$ " represent the results of using autoCenter.

offset tests and typically by a factor of 1000 in the large pixel offset tests.

Figure 2 shows a fair amount of scatter in the corrected dynamic range achieved. We believe this to be the result of residual errors in the centroiding as offsets on two axes produced lower dynamic range than comparable offsets on a single axis. All corrected dynamic range values were substantially better than that obtained using a 0.01 pixel offset without correction. At very high dynamic range the accuracy of the centroiding needs to be exceedingly precise. This is possible in this test as there are no systematic errors and the model is exactly a point source. In the real sky, resolution may be a problem even for a source whose size is a very small fraction of the PSF; position errors of less than 0.0025 of the PSF seem to be capable of limiting dynamic range so resolution on similar scales might be a problem. In Fig. 3 the efficacy of the correction seems to diminish with increasing pixel offset. This may be as much a problem with the simulated data as with the imaging; the larger position shifts needed to model a source with a substantial offset from the pointing center will result in loss of numerical precision.

Non co-planar effects coupling to the pixelization can limit significantly the dynamic range achieved. A source observed $20 \%$ of the way to the edge of its imaging facet suffers comparable dynamic range loss to a central source observed 


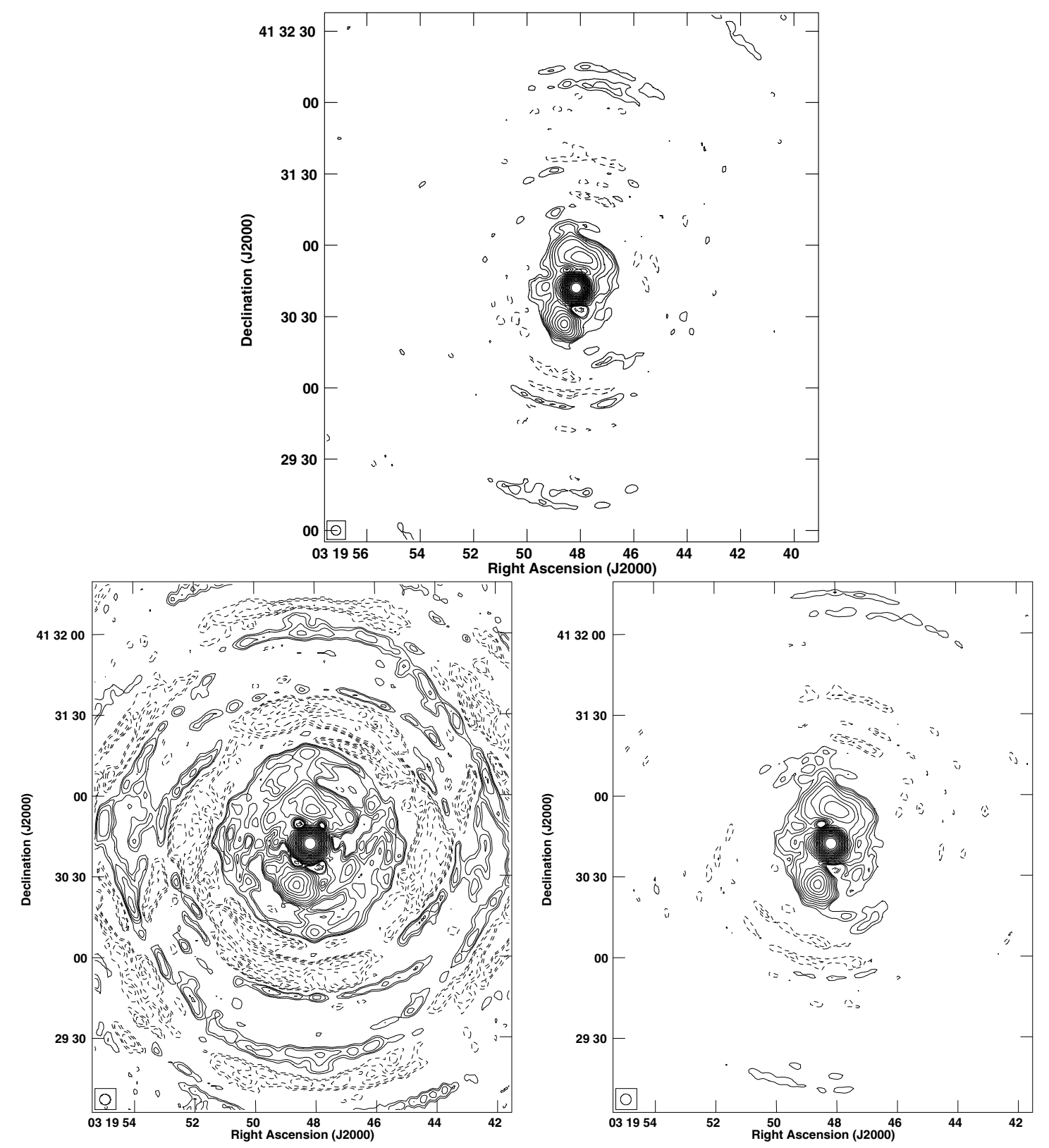

Fig. 4. 3C 84 with contour interval of powers of $\sqrt{2}$. The same contouring relative to the peak in the image is used for all plots. Top: $3 \mathrm{C} 84$ observed at the pointing center. The most negative contour (dashed) is at $-8 \mathrm{mJy} / \mathrm{beam}$. Bottom left: portion of wide-field image with $3 \mathrm{C} 84$ at the half power of the antenna pattern. The most negative contour (dashed) is at $-11 \mathrm{mJy} /$ beam. Bottom right: like Left except using the autoCenter technique. The most negative contour (dashed) is at $-4 \mathrm{mJy} / \mathrm{beam}$.

0.2 cells from the closest pixel. This indicates that imaging using the Fly's Eye technique needs to be applied with bright sources at the center of a facet (tangent point) and not merely on a pixel. A coarser grid spacing will likely lead to larger errors than presented here.

\subsection{Actual data}

A test using real data and wide-field imaging was made using the VLA at $1.4 \mathrm{GHz}$ and observations of $3 \mathrm{C} 84$ (peak $=24.6 \mathrm{Jy}$ ). $3 \mathrm{C} 84$ was observed both at the pointing center and at the half power point of the beam. The observations were made in spectral mode with 15 channels of $390 \mathrm{kHz}$ bandwidth. The data were bandpass calibrated in addition to the amplitude and phase calibration and the edge channels were excluded from processing.
Wide-field $3671 \times 3671$ pixel images were made to cover the primary beam of the antennas using the Fly's Eye technique and a 37 facet mosaic. In all cases, Obit task Imager determined and applied amplitude and phase self-calibration to optimize the dynamic range. The data set with $3 \mathrm{C} 84$ at the half power of the antenna power pattern was imaged both with and without the autoCenter technique. In these images, $3 \mathrm{C} 84$ was half-way to the edge of its facet (co-planarity limit) and 0.4 of a cell from the nearest grid cell in Right Ascension and 0.3 of a cell in Declination. Sampling was 4 pixels per beam. Contour plots of a portion of the images around 3C 84 are shown in Fig. 4.

It is immediately obvious from Fig. 4 that the autoCenter technique helps improve the dynamic range of the image with $3 \mathrm{C} 84$ well away from the pointing center. This is explored further in Table 2 which gives the relevant image statistics. The rms was determined in $601 \times 601$ pixel windows either centered on 
Table 2. 3C 84 dynamic range.

\begin{tabular}{|c|c|c|c|c|c|}
\hline$\overline{\text { Image }}$ & $\begin{array}{r}\text { Peak } \\
\text { Jy }\end{array}$ & $\begin{array}{r}\text { Near rms } \\
\text { Jy }\end{array}$ & $\begin{array}{r}\text { Far rms } \\
\text { Jy }\end{array}$ & Near DR & $\overline{\overline{\text { Far DR }}}$ \\
\hline Center $^{1}$ & 24.6 & 0.0020 & 0.00122 & 12289 & 20145 \\
\hline Half $^{2}$ & 12.0 & 0.0024 & 0.00121 & 5029 & 9933 \\
\hline Half/auto ${ }^{3}$ & 11.6 & 0.0010 & 0.00055 & 11745 & 21163 \\
\hline
\end{tabular}

Notes: ${ }^{1}$ 3C 84 observed at pointing center; ${ }^{2} 3 \mathrm{C} 84$ observed at half power; ${ }^{3}$ C 84 observed at half power using autoCenter.

3C 84 ("Near rms") or far from 3C 84 ("Far rms"). The near rms values were determined using a histogram analysis.

As can be seen from Table 2, using the autoCenter technique doubles the effective dynamic range of the image, even in regions far from the obvious artifacts caused by 3C 84 and does even better near the source. The autoCenter image has comparable dynamic range to the observation with $3 \mathrm{C} 84$ on axis. Especially in the neighborhood of $3 \mathrm{C} 84$, it is clear that other systematics are limiting the dynamic range. Indeed, given the extended emission surrounding 3C 84 the images are limited by uv-coverage as the test observations lacked the necessary short spacings. We find that this limitation is more severe than other systematic effects such as bandpass mismatches, pointing errors and beam squint. We have re-imaged the data after discarding baselines shorter than $12 \mathrm{k} \lambda$ and we have obtained a dynamic range that is $\sim 20 \%$ higher (again using the autoCenter technique) although the extended flux that surrounds the core of $3 \mathrm{C} 84$ is, of course, lost to the baseline restriction.

The difference in execution times for processing with and without the autoCenter technique depends on the details, i.e., number of self-calibrations, structure in the field, etc.; but is seldom significant. The cost of the extra, shallow CLEAN to locate sources to be re-centered can be partially or totally compensated by less time spent modeling artifacts in subsequent CLEANs. In the test presented in this section, processing without autoCentering actually took $1 \%$ longer than with autoCentering.

\section{Conclusion}

We have demonstrated that the dynamic range obtained from imaging interferometric observations can be adversely affected by pixelization of the images. Image pixelization effects can limit dynamic range to about $10^{4}$ even for point sources and non co-planar effects can limit the dynamic range to about $10^{3}$ even with a moderately conservative limit on facet size. The higher dynamic range needed for the EVLA and LOFAR, where wide fields of view with numerous bright sources will be common, need improved techniques to circumvent these limits.

The autoCenter technique presented here has shown factors of 50 to 1000 improvement in images made from simulated data with no noise or systematic errors. An improvement greater than a factor of 2 was achieved in images made from real observations of the bright source $3 \mathrm{C} 84$, even far from the obvious artifacts due to the source. Due to the lower level of artifacts, processing using this technique on the 3C 84 test presented above used marginally less computer time than without.

Fractional pixel corrections are more difficult to implement in a single image $w$-projection method. A simple shift can center a single source, also varying the pixel spacing could center two sources and a rotation could add a third but centering a larger number of sources will not be possible. The introduction of separate, simultaneous " $w$-projection" grids to accommodate such strong sources would seem to reduce the benefits of the " $w$-projection" technique ${ }^{2}$. Even grid-less subtraction of strong sources will require some shifting of their cataloged position due to foreground and (variable) instrumental effects. One such procedure discussed in the literature (Voronkov \& Wieringa 2004) seems to be much more computationally expensive than the procedure discussed in this paper, even when dealing with a small number of sources.

The technique described here can be applied to an arbitrary number of point-like sources. The tests presented here suggest that high dynamic range imaging of bright extended sources needs a better set of basis functions than the delta functions on grid cells that are used by CLEAN.

Acknowledgements. The authors would like to thank Huib Intema of Leiden University for stimulating discussions on this subject. We would also like to thank the anonymous referee for useful comments leading to an improved presentation of this technique.

\section{References}

Bennett \& WMAP team 2007, NASA Web site, http: //lambda.gsfc . nasa. gov/product/map/dr2/-ptsrc_catalog_get.cfm

Briggs, D. S., \& Cornwell, T. J. 1992, in Astronomical Data Analysis Software and Systems I, ed. D. M. Worrall, C. Biemesderfer, \& J. Barnes, ASP Conf. Ser., 25, 170

Condon, J. J., Cotton, W. D., Greisen, E. W., et al. 1998, AJ, 115, 1693

Cornwell, T. J., Golap, K., \& Bhatnagar, S. 2005, in Astronomical Data Analysis Software and Systems XIV, ed. P. Shopbell, M. Britton, \& R. Ebert, ASP Conf. Ser., 347, 86

Cornwell, T. J., \& Perley, R. A. 1992, A\&A, 261, 353

Cotton, W. D. 1989, in Synthesis Imaging in Radio Astronomy, ed. R. A. Perley, F. R. Schwab, \& A. H. Bridle, ASP Conf. Ser., 6, 243

Cotton, W. D. 2008, PASP, 120, 439

Perley, R. A. 1999a, in Synthesis Imaging in Radio Astronomy II, ed. G. B. Taylor, C. L. Carilli, \& R. A. Perley, ASP Conf. Ser., 180, 275

Perley, R. A. 1999b, in Synthesis Imaging in Radio Astronomy II, ed. G. B. Taylor, C. L. Carilli, \& R. A. Perley, ASP Conf. Ser., 180, 383

Schwab, F. R. 1983, in Indirect Imaging, Measurement and Processing for Indirect Imaging (Australia, Cambridge, England, New York: Cambridge University Press), 333

Thompson, A. R. 1999, in Synthesis Imaging in Radio Astronomy II, ed. G. B. Taylor, C. L. Carilli, \& R. A. Perley, ASP Conf. Ser., 180, 11

Voronkov, M. A., \& Wieringa, M. H. 2004, Exp. Astr., 18, 13

\footnotetext{
2 Alternatively, a finite number of tangent facets could be used to supplement the " $w$-projection" grid and "unboxes" used in a manner similar to the implementation discussed here. Again, this would seem to reduce the benefits of the " $w$-projection" technique.
} 